\title{
Beyond platelet functional testing: value in the aggregate
}

\begin{abstract}
"...one looks forward to the further evolution of standardized point-of-care testing that might add, for example, a more global test of hemostatic status to allow finer tuning of antithrombotic cocktails that will, no doubt, be the future of antithrombotic therapy."
\end{abstract}

\section{KEYWORDS: ADP $\approx$ clopidogrel $\approx$ genomics $\approx$ metabolism $\approx \mathrm{P}_{12} \approx$ pharmacokinetics - platelet testing $=$ prasugrel $\|$ thienopyridines}

In a review of functional testing methods for the antiplatelet effect of $\mathrm{P} 2 \mathrm{Y}_{12}$ antagonists, featured in this issue of Biomarkers in Medicine, Price and Barker succinctly summarize several pharmacodynamic $(\mathrm{PD})$ tests of platelet reactivity/function, namely light transmission aggregometry (LTA), vasodilator-stimulated phosphoprotein (VASP), VerifyNow ${ }^{\circledR} \mathrm{P} 2 \mathrm{Y} 12$ and multiple electrode platelet aggregometry [1]. The article largely focuses on the application of these PD assays to the evaluation of a registered antiplatelet agent, clopidogrel, in the postapproval clinical arena. An important finding that has been established utilizing these tests is the substantial interindividual variation in the platelet inhibitory effect of clopidogrel and the resultant consequences on clinical outcome. Beyond clopidogrel, these, and alternative assays, have played an important role in the identification and preclinical and clinical pharmacological evaluation of novel antiplatelet agents. Recent reports on ticagrelor and elinogrel, novel direct-acting $\mathrm{P} 2 \mathrm{Y}_{12}$ antagonists, have utilized the aforementioned assays to provide important data on the PD profile of these agents compared with clopidogrel $[2,3]$. Similarly, platelet function testing, supplemented by a variety of complementary assays, has played a critical role in the identification and clinical development of prasugrel, a thirdgeneration thienopyridyl $\mathrm{P}_{2} \mathrm{Y}_{12}$ antagonist [4-6]. Prasugrel, like clopidogrel, is a prodrug that must be converted to an active metabolite (AM) that binds to and inhibits platelet $\mathrm{P} 2 \mathrm{Y}_{12} \mathrm{ADP}$ receptors and, therefore, inhibits ADP-mediated platelet aggregation, and a variety of other prothrombotic and proinflammatory activities [7].

Early preclinical evaluation of prasugrel using LTA documented that it provides approximately equivalent levels of platelet inhibition as clopidogrel but at a tenth of the dose [4]. Numerous subsequent LTA studies performed in healthy subjects and in patients with cardiovascular disease confirmed this lower dose requirement in humans [5]. In vitro evaluation of clopidogrel's and prasugrel's AMs using LTA demonstrated them to have similar inherent activity. Therefore, the difference in administered dose-response between prasugrel and clopidogrel was presumably associated with the level of in vivo exposure of platelets to the AMs. With the development of assays to measure the plasma concentrations (pharmacokinetics [PK]) of the AMs, it was confirmed that there was indeed a 12-14-fold greater exposure to prasugrel's AM, as measured by maximum drug concentration and area under the curve, than to clopidogrel's, translating to an approximately 60-fold increment on a dose-adjusted basis [5]. This difference in exposure reflects the presence of an ester moiety in clopidogrel, which is rapidly and extensively $(\sim 85 \%)$ hydrolyzed to an inactive metabolite upon administration, leaving only approximately $15 \%$ of the administered dose available for conversion to its AM. Conversion of this remaining intact clopidogrel requires two cytochrome P450 (CYP) oxidative steps, mediated by CYP3A4, CYP2B6, CYP2C19, CYP2C9 and CYP1A2 [7]. Prasugrel also contains an ester moiety that is rapidly hydrolyzed. However, in the case of prasugrel, the location of the ester moiety is such that its hydrolysis product does not enter a redundant pathway, but actually provides the substrate for a single-step conversion to the AM. This single-step conversion can be mediated by several alternative CYPs, including CYP3A4, CYP2B6, CYP2C9 and CYP2C19 [8]. Taken in aggregate, this series of metabolic, PK and PD studies provided a mechanistic basis for the increased exposure to prasugrel's AM, and more rapid and consistent inhibition of platelet

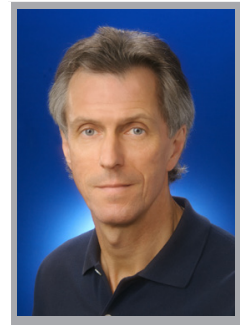


function compared with that found with clopidogrel. For this reason, it can perhaps be appreciated that an integrated approach to drug characterization, in other words, one that includes PK, PD and metabolic evaluation, can provide a much greater understanding of a molecule's actions than platelet PD testing alone, and when such assays are applied early in a clinical development program, can greatly aid in subsequent clinical trial design/dose selection.

This comprehensive understanding of the factors that differentiated prasugrel from clopidogrel encouraged further clinical evaluation and prompted broader platelet functional testing to compare prasugrel with clopidogrel, and incorporated use of the (then) emerging assays of VASP and VerifyNow P2Y12. While differentiating prasugrel from clopidogrel, it was noted that, compared with LTA, these two newer assays demonstrated a relative loss of sensitivity at high and low levels of inhibition $[9,10]$. However, for both assays, the emerging target threshold values for efficacy (reduced ischemic events) with clopidogrel are within the linear portion of the relationship. Interestingly, threshold values for safety (bleeding) have been elusive with these tests [11]. Whether this reflects a lack of dynamic range at high levels of inhibition and/or that the studies have been underpowered for this end point is not clear.

\section{"Given the value of incorporating PK, PD and genetic studies into clinical trials, one might consider their value in drug/dose selection in routine clinical practice."}

Phase Ib and II dose-ranging studies provided the basis for dose selection for the Phase III evaluation of the efficacy and safety of prasugrel. In a Phase Ib dose-ranging study in cardiovascular patients, it was determined, using LTA, that a loading dose (LD) of $60 \mathrm{mg}$ prasugrel resulted in more rapid and greater inhibition of aggregation than a $300 \mathrm{mg}$ LD of clopidogrel, and that of four escalating daily maintenance doses (MDs) of prasugrel studied $(5,7.5,10$ and $15 \mathrm{mg}$ ), $10 \mathrm{mg}$ was the minimal dose that provided consistently greater and less variable inhibition of platelet aggregation than $75 \mathrm{mg}$ of clopidogrel, but avoided the higher levels of inhibition found with the $15 \mathrm{mg}$ dose [12]. Trial to Assess Improvement in Therapeutic Outcomes by Optimizing Platelet Inhibition with Prasugrel (TRITON)-Thrombolysis in Myocardial Infarction (TIMI) 38, a 13,608 patient study that compared the efficacy and safety of prasugrel in acute coronary syndrome (ACS) patients undergoing percutaneous coronary intervention demonstrated that the $60 \mathrm{mg} \mathrm{LD} / 10 \mathrm{mg} \mathrm{MD}$ doses provided superior protection against ischemic events than a standard dose of clopidogrel. Prasugrel was however associated with a greater rate of TIMI noncoronary artery bypass graft major bleeding [13].

During the conduct of TRITON, with the growing awareness of clopidogrel's variable antiplatelet effects, the use of $600 \mathrm{mg}$ LDs of clopidogrel became commonplace. Platelet function testing, using many of the assays described by Price and Barker [1], were employed in several studies that compared prasugrel with high-dose clopidogrel, and it was documented that, despite a comparison with double the approved LD and MD of clopidogrel, greater and more consistent platelet inhibition was achieved with prasugrel $[9,14,15]$. Platelet function testing utilizing LTA, VASP and VerifyNow P2Y12 was also employed to ensure that there were no apparent safety issues when switching directly from clopidogrel to prasugrel without a washout period, and that prasugrel provided similar levels of platelet inhibition to that found when prasugrel was administered to clopidogrel-naive patients $[10,15]$. The former study illustrates the striking agreement between results obtained with VerifyNow P2Y12 and LTA [10].

Substudies that were nested within the TRITON trial included platelet PD, PK and genetic assessments. These substudies provided valuable information regarding the relative performance of prasugrel versus clopidogrel, with the platelet PD substudy confirming, in a real-world population of ACS patients undergoing percutaneous coronary intervention, that prasugrel provided greater platelet inhibition than clopidogrel as measured by LTA and VASP [16]. The genetic substudy provided clinical confirmation of concepts that had emerged from earlier data that pointed to CYP2C19 loss-of-function alleles, leading to impaired AM generation and attenuated inhibition of platelet aggregation for clopidogrel but not prasugrel. The TRITON efficacy data in clopidogrel-treated patients carrying at least one of the loss-of-function alleles had a poorer outcome than those who were not carriers [17]. By contrast, CYP2C19 status had no influence on outcomes in prasugrel-treated patients [18]. Subsequent analysis has recently identified additional genes associated with poor outcome on clopidogrel [19]. The PK substudy in TRITON allowed for an evaluation of the relationship between exposure to prasugrel and 
efficacy and bleeding. It was noted that two populations in which TIMI noncoronary artery bypass graft major bleeding was more common had a greater estimated exposure to prasugrel's AM than the overall population [20]. These two populations were those with low body weight $(<60 \mathrm{~kg})$ and advanced age ( $\geq 75$ years). These data provided a basis for the recommendations for a reduced prasugrel MD of $5 \mathrm{mg}$ in these two populations. Ongoing follow-up clinical studies are formally evaluating the efficacy and safety of $5 \mathrm{mg}$ prasugrel in these populations, and include extensive PK and platelet PD assessments. The ease of use of VerifyNow P2Y12 has enabled broad-based PD testing in the ongoing Phase III Targeted Platelet Inhibition to Clarify the Optimal Strategy to Medically Manage ACS (TRILOGY-ACS) study in medically managed ACS patients, which includes both 5 and $10 \mathrm{mg}$ doses of prasugrel.

\section{"There are pros and cons for both PD and genetic testing in guiding the choice of $P 2 Y_{12}$ antagonist and dose thereof, but one has to acknowledge that the advantages of functional testing outweigh those of genetic testing."}

Given the value of incorporating PK, PD and genetic studies into clinical trials, one might consider their value in $\mathrm{drug} / \mathrm{dose}$ selection in routine clinical practice. At present, evaluation of systemic exposure to thienopyridine metabolites is not practical on a large scale. There are pros and cons for both PD and genetic testing in guiding the choice of $\mathrm{P}_{2} \mathrm{Y}_{12}$ antagonist and dose thereof, but one has to acknowledge that the advantages of functional testing outweigh those of genetic testing. While genetic testing can be performed at any time (on or off drug therapy), the result, which can currently take several days to be obtained, may be limited to a single gene. As such, the result may not account for the presence of polymorphisms in several other genes that have been implicated in clopidogrel's activity $(A B C B-1$, esterases and $P O N-1$ ) and, likewise, do not take into account other factors that may upregulate (e.g., herbal supplements, smoking and comedications) or downregulate/inhibit CYP activity (e.g., proton-pump inhibitors and azole antifungals). There are also numerous other intrinsic and extrinsic factors that have been associated with altered thienopyridine activity [21]. By contrast, by assessing platelet reactivity by functional PD assays, all of the known (and unknown) variables that influence the net platelet response/reactivity will be, by default, accounted for. However, the subject needs to be taking the drug at the time of testing. This is not to say that functional testing is without its limitations, and one looks forward to the further evolution of standardized point-ofcare testing that might add, for example, a more global test of hemostatic status to allow finer tuning of antithrombotic cocktails that will, no doubt, be the future of antithrombotic therapy.

\section{Financial \& competing interests disclosure}

JA Jakubowski is an employee and stockholder of Eli Lilly and Company who market prasugrel under the trade name Efient ${ }^{\circledR}$. The author has no other relevant affliations or financial involvement with any organization or entity with a financial interest in or financial conflict with the subject matter or materials discussed in the manuscript apart from those disclosed.

No writing assistance was utilized in the production of this manuscript.

\section{Bibliography}

1 Price MJ, Barker CM: Functional testing methods for the antiplatelet effect of $\mathrm{P} 2 \mathrm{Y}_{12}$ receptor antagonists. Biomarkers Med. 5(1), 43-51 (2011).

2 Gurbel PA, Bliden KP, Butler K et al: Randomized double-blind assessment of the ONSET and OFFSET of the antiplatelet effects of ticagrelor versus clopidogrel in patients with stable coronary artery disease: the ONSET/OFFSET study. Circulation 120, 2577-2585 (2009).

3 Gurbel PA, Bliden KP, Antonino MJ et al: : The effect of elinogrel on high platelet reactivity during dual antiplatelet therapy and the relation to $C Y P 2 C 19^{*} 2$ genotype: first experience in patients. J. Thromb. Haemost. 8, 43-53 (2010).
4 Niitsu Y, Jakubowski JA, Sugidachi A, Asai A: Pharmacology of CS-747 (prasugrel, LY640315), a novel, potent antiplatelet agent with in vivo $\mathrm{P}_{2} \mathrm{Y}_{12}$ receptor antagonist activity. Semin. Thromb. Hemost. 31, 184-194 (2005).

5 Jakubowski JA, Winters KJ, Naganuma H, Walletin L: Prasugrel: a novel thienopyridine antiplatelet agent. A review of preclinical and clinical studies and the mechanistic basis for its distinct antiplatelet profile. Cardiovas. Drug Rev. 25, 358-374 (2007).

6 Jakubowski JA, Riesmeyer JS, Close SL, Leishman AG, Erlinge D: TRITON and beyond: new insights into the profile of prasugrel. Cardiovasc. Ther. 29 (2011) (In Press).
7 Frelinger AL, Jakubowski JA, Li Y et al:: The active metabolite of prasugrel inhibits ADP-stimulated thrombo-inflammatory markers of platelet activation: influence of other blood cells, calcium, and aspirin. Thromb. Haemost. 98, 192-200 (2007).

8 Farid NA, Kurihara A, Wrighton SA: Metabolism and disposition of the thienopyridine antiplatelet drugs ticlopidine, clopidogrel, and prasugrel in humans. J. Clin. Pharmacol. 50, 126-142 (2010).

9 Jakubowski JA, Payne CD, Li YG et al.: A comparison of the antiplatelet effects of prasugrel and high-dose clopidogrel as assessed by VASP-phosphorylation and light transmission aggregometry. Thromb. Haemost. 99, 215-222 (2007). 
10 Jakubowski JA, Payne CD, Li YG et al:: The use of the VerifyNow P2Y12 point-of-care device to monitor platelet function across a range of $\mathrm{P} 2 \mathrm{Y}_{12}$ inhibition levels following prasugrel and clopidogrel administration. Thromb. Haemost. 99, 409-415 (2008).

11 Breet NJ, van Werkum JW, Bouman HJ et al.: Comparison of platelet function tests in predicting clinical outcome in patients undergoing coronary stent implantation. JAMA 303, 754-762 (2010).

12 Jernberg T, Payne CD, Winters KJ et al.: Prasugrel achieves greater inhibition of platelet aggregation and a lower rate of non-responders compared with clopidogrel in aspirin-treated patients with stable coronary artery disease. Eur. Heart J. 27, 1166-1173 (2006).

13 Wiviott SD, Braunwald E, McCabe $\mathrm{CH}$ et al.: Prasugrel versus clopidogrel in patients with acute coronary syndromes. N. Engl. J. Med. 357, 2001-2015 (2007).

14 Wiviott SD, Trenk D, Frelinger AL et al. Prasugrel compared with high loading-and maintenance-dose clopidogrel in patients with planned percutaneous coronary intervention: The PRINCIPLE-TIMI 44 trial. Circulation 116, 2923-2932 (2007).

15 Angiolillo DJ, Saucedo JF, Deraad R et al.; SWAP Investigators: Increased platelet inhibition after switching from maintenance clopidogrel to prasugrel in patients with acute coronary syndromes: results of the SWAP (Switching Anti Platelet) study. J. Am. Coll. Cardiol. 56, 1017-1023 (2010).

16 Michelson AD, Frelinger AL III, Braunwald E et al:: Pharmacodynamic assessment of platelet inhibition by prasugrel vs. clopidogrel in the TRITON-TIMI 38 trial. Eur. Heart J. 30 , 1753-1763 (2009).

17 Mega JL, Close SL, Wiviott SD et al.: Cytochrome P-450 polymorphisms and response to clopidogrel. N. Engl. J. Med. 360, 354-362 (2009).

18 Mega JL, Close SL, Wiviott SD et al: Cytochrome $\mathrm{P} 450$ genetic polymorphisms and the response to prasugrel: relationship to pharmacokinetic, pharmacodynamic, and clinical outcomes. Circulation 119, 2553-2560 (2009).

19 Mega JL, Close SL, Wiviott SD et al.: Genetic variants in $A B C B 1$ and CYP2C19 and cardiovascular outcomes after treatment with clopidogrel and prasugrel in the TRITON-TIMI 38 trial: a pharmacogenetic analysis. Lancet 376, 1312-1319 (2010).

20 Wrishko RE, Ernest CS 2nd, Small DS et al.: Population pharmacokinetic analyses to evaluate the influence of intrinsic and extrinsic factors on exposure of prasugrel active metabolite in TRITON-TIMI 38. J. Clin. Pharmacol. 49, 984-998 (2009).

21 Small DS, Farid NA, Payne CD et al: Effect of intrinsic and extrinsic factors on the clinical pharmacokinetics and pharmacodynamics of prasugrel. Clin. Pharmacokinet. 49, 777-798 (2010). 